\title{
Crystal structure of a 6.5 MDa bacterial microcompartment shell
}

\author{
Markus Sutter ${ }^{1,2}$, Basil Greber ${ }^{2,3}$, Clement Aussignargues ${ }^{1}$, Cheryl A. Kerfeld ${ }^{1,2,4^{*}}$ \\ ${ }^{1}$ MSU-DOE Plant Research Laboratory, Michigan State University, East Lansing, MI 48824, USA. \\ ${ }^{2}$ Molecular Biophysics and Integrated Bioimaging Division, Lawrence Berkeley National Laboratory, \\ Berkeley, CA 94720, USA. \\ ${ }^{3}$ California Institute for Quantitative Biosciences (QB3), University of California, Berkeley, California \\ 94720, USA.
}

${ }^{4}$ Department of Biochemistry and Molecular Biology, Michigan State University, East Lansing, MI 48824, USA.

Many bacteria contain self-assembling organelles composed entirely of protein. These bacterial microcompartments consist of an enzymatic core encapsulated by a selectively permeable protein shell. The best known example is the carboxysome for $\mathrm{CO}_{2}$ fixation, and there are a number of microcompartments that are involved in degradation of various organic compounds. The shell sequesters enzymatic reactions from the cytosol to prevent undesired side reactions and protect the cell from toxic intermediates. There is a wealth of structural information for single building blocks but the principles of shell assembly have remained elusive. We present the X-ray crystal structure of an intact shell from Haliangium ochraceum at a resolution of $3.5 \AA$, revealing the basic principles of bacterial microcompartment shell construction. Half of the particle with icosahedral symmetry is found in the asymmetric part of the $C 222_{1}$ unit cell with dimensions of $394 \times 638 \times 642 \AA$. The interactions we see in the crystal structure are mediated by highly conserved and are likely to apply to all functionally diverse organelles and can inform the design and engineering of shells with new functionalities.

Sutter, M., B. Greber, C. Aussignargues and C. A. Kerfeld (2017). "Assembly principles and structure of a 6.5-MDa bacterial microcompartment shell." Science 356(6344): 1293-1297. 